\title{
An asymptotically sharp coefficients estimate for harmonic $K$-quasiconformal mappings
}

Hong-Ping Li*

\section{"Correspondence:}

Ihp306@hqu.edu.cn

School of Mathematical Sciences,

Huagiao University, Quanzhou,

362021, China

\section{Springer}

\begin{abstract}
By using the improved Hübner inequalities, in this paper we obtain an asymptotically sharp lower bound estimate for the coefficients of harmonic K-quasiconformal self-mappings of the unit disk $\mathbb{D}$ which keep the origin fixed. The result partly improves the former results given by (Partyka and Sakan in Ann. Acad. Sci. Fenn., Math. 30:167-182, 2005) and (Zhu and Zeng in J. Comput. Anal. Appl. 13:1081-1087, 2011). Furthermore, using some estimate for the derivative of the boundary function of a harmonic K-quasiconformal self-mapping $w$ of $\mathbb{D}$ which keeps the origin fixed, we obtain an upper bound estimate for the coefficients of $W$.
\end{abstract}

MSC: Primary 30C62; secondary 30C20; 30F15

Keywords: Heinz inequality; Hübner inequalities; coefficients estimate; harmonic quasiconformal mapping

\section{Introduction}

Let $\mathbb{D}=\{z:|z|<1\}$ denote the unit disk, $w(z)$ be a harmonic mapping defined in $\mathbb{D}$. Then $w(z)$ can be presented as $w(z)=h(z)+\overline{g(z)}$, where

$$
h(z)=\sum_{n=0}^{\infty} a_{n} z^{n} \quad \text { and } \quad g(z)=\sum_{n=1}^{\infty} b_{n} z^{n}
$$

are both analytic in $\mathbb{D}$. By Lewy's theorem [3], we know that $w(z)$ is locally univalent and sense-preserving in $\mathbb{D}$ if and only if its Jacobian satisfies the following inequality:

$$
J_{f}(z)=\left|w_{z}(z)\right|^{2}-\left|w_{\bar{z}}(z)\right|^{2}=\left|h^{\prime}(z)\right|^{2}-\left|g^{\prime}(z)\right|^{2}>0
$$

for all $z \in \mathbb{D}$. One of the basic properties for harmonic self-mappings of $\mathbb{D}$ is the Heinz inequality [4].

Lemma A Let $w$ map the unit disk harmonically onto itself with $w(0)=0$. Then

$$
\left|w_{z}(0)\right|^{2}+\left|w_{\bar{z}}(0)\right|^{2} \geq c
$$

for some absolute constant $c>0$.

(c) 2016 Li. This article is distributed under the terms of the Creative Commons Attribution 4.0 International License (http://creativecommons.org/licenses/by/4.0/), which permits unrestricted use, distribution, and reproduction in any medium, provided you give appropriate credit to the original author(s) and the source, provide a link to the Creative Commons license, and indicate if changes were made. 
Subsequently, in 1982, Hall [5] obtained the sharp lower bound of $c$.

Theorem B Let $w(z)=h(z)+\overline{g(z)}=\sum_{n=1}^{\infty} a_{n} z^{n}+\overline{\sum_{n=1}^{\infty} b_{n} z^{n}}$ be a univalent harmonic mapping of the unit disk onto itself, then its coefficients satisfy the inequality

$$
\left|a_{1}\right|^{2}+\left|b_{1}\right|^{2} \geq \frac{27}{4 \pi^{2}}
$$

The lower bound $\frac{27}{4 \pi^{2}}$ is the best possible.

Let

$$
p(r, x-\varphi)=\frac{1-r^{2}}{2 \pi\left(1-2 r \cos (x-\varphi)+r^{2}\right)}
$$

denote the Poisson kernel, then every bounded harmonic mapping $w$ defined in $\mathbb{D}$ has the following representation:

$$
w(z)=P[f](z)=\int_{0}^{2 \pi} p(r, x-\varphi) f\left(e^{i x}\right) d x,
$$

where $z=r e^{i \varphi} \in \mathbb{D}$ and $f$ is a bounded integrable function defined on the unit circle $\mathbf{T}:=$ $\partial \mathbb{D}$.

Suppose that $w(z)$ is a sense-preserving univalent harmonic mapping of $\mathbb{D}$ onto a domain $\Omega \subseteq \mathbb{C}$. Then $w(z)$ is a harmonic $K$-quasiconformal mapping if and only if

$$
K(w):=\sup _{z \in \mathbb{D}} \frac{\left|w_{z}(z)\right|+\left|w_{\bar{z}}(z)\right|}{\left|w_{z}(z)\right|-\left|w_{\bar{z}}(z)\right|} \leq K .
$$

Under the additional assumption that $w(z)$ is a $K$-quasiconformal mapping, in 2005 Partyka and Sakan [1] obtained an asymptotically sharp variant of Heinz's inequality as follows (see also [2]).

Theorem $\mathbf{C}$ Let $w(z)$ be a harmonic $K$-quasiconformal mapping of $\mathbb{D}$ onto itself satisfying $w(0)=0$. Then the inequality

$$
\left|\partial_{z} w(z)\right|^{2}+\left|\partial_{\bar{z}} w(z)\right|^{2} \geq \frac{1}{4}\left(1+\frac{1}{K}\right)^{2} \max \left\{\frac{4}{\pi^{2}}, L_{K}^{2}\right\}
$$

holds for every $z \in \mathbb{D}$, where

$$
L_{K}:=\frac{2}{\pi} \int_{0}^{\frac{1}{\sqrt{2}}} \frac{d\left(\Phi_{1 / K}(s)^{2}\right)}{s \sqrt{1-s^{2}}}
$$

is a strictly decreasing function of $K$. For $L>0, \Phi_{L}(s)$ is the Hersch-Pfluger distortion function defined by the equalities $\Phi_{L}(s):=\mu^{-1}(\mu(s) / L), 0<s<1 ; \Phi_{L}(0):=0, \Phi_{L}(1):=1$, where $\mu(s)$ stands for the module of Grötzsch's extremal domain $\mathbb{D} \backslash[0, s]$.

In 2010, Qiu and Ren [6] improved the Hübner inequalities as follows. 
Theorem D For all $s \in(0,1)$ and $K \in(1, \infty)$, we have

$$
4^{1-K} s^{K} \leq \Phi_{1 / K}(s)<4^{D(s)(1-K)} s^{K}
$$

and

$$
s^{1 / K} \leq \Phi_{K}(s)<4^{\left(1-s^{2}\right)^{\frac{3}{4}}(1-1 / K)} s^{1 / K},
$$

where $D(s)=(1-s)(1+s)^{1 / \ln 4}$.

A sense-preserving harmonic mapping of $\mathbb{D}$ onto itself can be represented as the Poisson extension of the boundary function $f\left(e^{i t}\right)=e^{i \gamma(t)}$, where $\gamma(t)$ is a continuous nondecreasing function with $\gamma(2 \pi)-\gamma(0)=2 \pi$ and $\gamma(t+2 \pi)=\gamma(t)+2 \pi(c f$. [7, 8]). The coefficients $a_{n}$ and $b_{n}$ have an alternative interpretation as Fourier coefficients of the periodic function $e^{i \gamma(t)}$, and so Heinz's lemma can be viewed as a statement about Fourier series.

In this paper, assuming that $w(z)$ is a harmonic $K$-quasiconformal mapping of $\mathbb{D}$ onto itself satisfying $w(0)=0$, by using Theorem $\mathrm{D}$ we obtain a sharp lower bound for its coefficients as follows:

$$
\left|a_{1}\right|^{2}+\left|b_{1}\right|^{2} \geq B_{1}(K):=2-2^{2(1-1 / K)\left(2+2^{5 / 4}\right)} \frac{2 K^{2} \Gamma\left(\frac{2}{K}\right)}{(K+1) \Gamma^{2}\left(\frac{1}{K}\right)}
$$

which satisfies $\lim _{K \rightarrow 1^{+}} B_{1}(K)=1$, where $\Gamma$ is the gamma function.

For $n \geq 2$ we have

$$
\left|a_{n}\right|^{2}+\left|b_{n}\right|^{2} \geq B_{n}(K)
$$

where

$$
\begin{aligned}
& B_{n}(K):=\chi(K)+\frac{2^{2(1-1 / K)\left(2+2^{5 / 4}\right)} \Gamma\left(1+\frac{2}{K}\right)\left(n-1-\frac{1}{K}\right) !}{\Gamma^{2}\left(\frac{1}{K}\right)\left(n+\frac{1}{K}\right) \frac{1}{K}\left(n-1+\frac{1}{K}\right) !}, \\
& \left(n-1-\frac{1}{K}\right) !:=\left(n-1-\frac{1}{K}\right)\left(n-2-\frac{1}{K}\right) \cdots\left(1-\frac{1}{K}\right), \\
& \left(n-1+\frac{1}{K}\right) !:=\left(n-1+\frac{1}{K}\right)\left(n-2+\frac{1}{K}\right) \cdots\left(1+\frac{1}{K}\right),
\end{aligned}
$$

and

$$
\chi(K):=2-\frac{2^{2(1-1 / K)\left(2+2^{5 / 4}\right)} \Gamma\left(1+\frac{2}{K}\right)}{\Gamma^{2}\left(1+\frac{1}{K}\right)}
$$

is a decreasing function of $K$ with $\chi(1)=0$.

Assume that $w(z)=P[f](z)$ is a harmonic $K$-quasiconformal mapping of $\mathbb{D}$ onto itself with the boundary function $f\left(e^{i t}\right)=e^{i \gamma(t)}$, satisfying $w(0)=0$. In Theorem 3.2 of [9], Partyka and Sakan proved that the following inequalities:

$$
\frac{2^{5\left(1-K^{2}\right) / 2}}{\left(K^{2}+K-1\right)^{K}} \leq\left|f^{\prime}(z)\right| \leq K^{3 K} 2^{5\left(K-\frac{1}{K}\right) / 2}
$$


hold for a.e. $z=e^{i t} \in \mathbf{T}$. Applying the above inequalities we obtain an upper bound for the coefficients of a harmonic $K$-quasiconformal self-mapping $w(z)$ of $\mathbb{D}$ satisfying $w(0)=0$ as follows:

$$
\left|a_{n}\right|^{2}+\left|b_{n}\right|^{2} \leq A_{n}(K):=\frac{16}{n^{2} \pi^{2}} K^{6 K} 2^{5(K-1 / K)} .
$$

Furthermore we show that (9) and (10) are sharp as $K \rightarrow 1$.

\section{Auxiliary results}

Lemma 1 Let $K>1$ be a constant. Then the equality

$$
\int_{0}^{\pi} \sin ^{\frac{2}{K}}(t) \cos (2 n t) d t=\frac{\pi}{4 \frac{1}{K}} \frac{(-1)^{n} \Gamma\left(1+\frac{2}{K}\right)}{\Gamma\left(1+\frac{1}{K}-n\right) \Gamma\left(1+\frac{1}{K}+n\right)}
$$

holds for all nonnegative integer numbers $n=0,1,2, \ldots$

Lemma 2 Let $\varphi(t):=\left|\cos \frac{t}{2}\right|^{\frac{3}{2}}+\left|\sin \frac{t}{2}\right|^{\frac{3}{2}}$, for any $t \in[0,2 \pi]$. Then

$$
\max _{0 \leq t \leq 2 \pi} \varphi(t)=\varphi\left(\frac{\pi}{2}\right)=\sqrt[4]{2}
$$

Lemma 3 Let $w=P[f](z)$ be a harmonic $K$-quasiconformal self-mapping of $\mathbb{D}$ with the boundary function $f\left(e^{i t}\right)=e^{i \gamma(t)}$. For every $z_{1}=e^{i(s+t)}, z_{2}=e^{i(s-t)} \in \mathbf{T}$, let $\theta=\gamma(s+t)-\gamma(s-t)$. Then $f\left(z_{1}\right)=e^{i \theta} f\left(z_{2}\right)$ and the inequalities

$$
2^{10-10 K} \sin ^{2 K}(t) \leq \sin ^{2}\left(\frac{\theta}{2}\right) \leq 2^{2(1-1 / K)\left(1+2^{5 / 4}\right)} \sin ^{2 / K}(t)
$$

hold for every $0 \leq s<2 \pi, 0 \leq t \leq \pi$.

Proof According to the quasi-invariance of the harmonic measure (see (1.9) in [1]), we have

$$
\Phi_{1 / K}\left(\cos \frac{t}{2}\right) \leq \cos \frac{\theta}{4} \leq \Phi_{K}\left(\cos \frac{t}{2}\right)
$$

for every $0 \leq s<2 \pi, 0 \leq t \leq \pi$, and $\theta=\gamma(s+t)-\gamma(s-t)$. Since $\Phi_{K}^{2}(x)+\Phi_{1 / K}^{2}\left(\sqrt{1-x^{2}}\right)=1$ holds for every $0 \leq x \leq 1$, this shows that

$$
\Phi_{1 / K}\left(\sin \frac{t}{2}\right) \leq \sin \frac{\theta}{4} \leq \Phi_{K}\left(\sin \frac{t}{2}\right)
$$

Using the Hübner inequalities, (7) and (8), we see that $4^{1-K} s^{K} \leq \Phi_{1 / K}(s)<4^{D(s)(1-K)} s^{K}$ and $s^{1 / K} \leq \Phi_{K}(s)<4^{\left(1-s^{2}\right)^{\frac{3}{4}}(1-1 / K)} s^{1 / K}$. Applying (18), (19), and the above two inequalities, we have

$$
\sin ^{2}\left(\frac{\theta}{2}\right) \geq 4 \Phi_{1 / K}^{2}\left(\sin \frac{t}{2}\right) \Phi_{1 / K}^{2}\left(\cos \frac{t}{2}\right) \geq 2^{10(1-K)} \sin ^{2 K} t
$$


and

$$
\sin ^{2}\left(\frac{\theta}{2}\right) \leq 4 \Phi_{K}^{2}\left(\sin \frac{t}{2}\right) \Phi_{K}^{2}\left(\cos \frac{t}{2}\right) \leq 2^{2(1-1 / K)\left\{2\left[\left|\cos \left(\frac{t}{2}\right)\right|^{\frac{3}{2}}+\left|\sin \left(\frac{t}{2}\right)\right|^{\frac{3}{2}}\right]+1\right\}} \sin ^{\frac{2}{K}}(t)
$$

By using Lemma 2 we see that

$$
\left|\cos \frac{t}{2}\right|^{\frac{3}{2}}+\left|\sin \frac{t}{2}\right|^{\frac{3}{2}} \leq \sqrt[4]{2}
$$

This implies that

$$
2^{10-10 K} \sin ^{2 K}(t) \leq \sin ^{2}\left(\frac{\theta}{2}\right) \leq 2^{2(1-1 / K)\left(1+2^{5 / 4}\right)} \sin ^{2 / K}(t)
$$

hold for every $0 \leq s<2 \pi, 0 \leq t \leq \pi$, and $\theta=\gamma(s+t)-\gamma(s-t)$.

This completes the proof.

\section{Main results}

Theorem 1 Given $K>1$, let $w(z)=P[f](z)=h(z)+\overline{g(z)}$ be a harmonic K-quasiconformal self-mapping of $\mathbb{D}$ satisfying $w(0)=0$ with the boundary function $f\left(e^{i t}\right)=e^{i \gamma(t)}$, where

$$
h(z)=\sum_{n=1}^{\infty} a_{n} z^{n} \text { and } g(z)=\sum_{n=1}^{\infty} b_{n} z^{n}
$$

are both analytic in $\mathbb{D}$. Then

$$
\left|a_{1}\right|^{2}+\left|b_{1}\right|^{2} \geq B_{1}(K)
$$

where $B_{1}(K)$ is given by (9) and satisfies $\lim _{K \rightarrow 1^{+}} B_{1}(K)=1$. For $n \geq 2$,

$$
\left|a_{n}\right|^{2}+\left|b_{n}\right|^{2} \geq B_{n}(K)
$$

where $B_{n}(K)$ is given by (11) and satisfies $\lim _{n \rightarrow \infty} \lim _{K \rightarrow 1^{+}} B_{n}(K)=0$.

Proof Since $w(z)=P[f](z)=\sum_{n=1}^{\infty} a_{n} z^{n}+\overline{\sum_{n=1}^{\infty} b_{n} z^{n}}$, using Parseval's relation (cf. [7]) we have

$$
\frac{1}{2 \pi} \int_{0}^{2 \pi} e^{i[\gamma(s+t)-\gamma(s-t)]} d s=\sum_{n=1}^{\infty}\left(\left|a_{n}\right|^{2} e^{2 i n t}+\left|b_{n}\right|^{2} e^{-2 i n t}\right)
$$

for arbitrary $t \in R$. Taking real parts, we arrive at the formula

$$
1-2 J(t)=\sum_{n=1}^{\infty}\left(\left|a_{n}\right|^{2}+\left|b_{n}\right|^{2}\right) \cos (2 n t)
$$

where

$$
J(t)=\frac{1}{2 \pi} \int_{0}^{2 \pi} \sin ^{2}\left(\frac{\gamma(s+t)-\gamma(s-t)}{2}\right) d s .
$$


Since $w(z)$ is a harmonic $K$-quasiconformal mapping, by Lemma 3 we have

$$
2^{10-10 K} \sin ^{2 K}(t) \leq J(t) \leq 2^{2(1-1 / K)\left(1+2^{5 / 4}\right)} \sin ^{2 / K}(t) .
$$

Hence $\left(\left|a_{n}\right|^{2}+\left|b_{n}\right|^{2}\right) \int_{0}^{\pi} \cos (2 n t)(1+\cos (2 n t)) d t=\int_{0}^{\pi}(1-2 J(t))(1+\cos (2 n t)) d t$. Using (15) we also obtain

$$
\begin{aligned}
\left|a_{n}\right|^{2}+\left|b_{n}\right|^{2} & =\frac{2}{\pi}\left(\pi-2 \int_{0}^{\pi} J(t)(1+\cos (2 n t)) d t\right) \\
& \geq \frac{2}{\pi}\left(\pi-2 \cdot 2^{2(1-1 / K)\left(1+2^{5 / 4}\right)} \int_{0}^{\pi} \sin ^{\frac{2}{K}}(t)(1+\cos (2 n t)) d t\right) \\
& =2-2^{2(1-1 / K)\left(2+2^{5 / 4}\right)} \frac{\Gamma\left(1+\frac{2}{K}\right)}{\Gamma^{2}\left(1+\frac{1}{K}\right)}-\frac{2^{2(1-1 / K)\left(2+2^{5 / 4}\right)}(-1)^{n} \Gamma\left(1+\frac{2}{K}\right)}{\Gamma\left(1+\frac{1}{K}-n\right) \Gamma\left(1+\frac{1}{K}+n\right)} \\
& :=\chi(K)+\frac{2^{2(1-1 / K)\left(2+2^{5 / 4}\right)}(-1)^{n+1} \Gamma\left(1+\frac{2}{K}\right)}{\Gamma\left(1+\frac{1}{K}-n\right) \Gamma\left(1+\frac{1}{K}+n\right)} .
\end{aligned}
$$

For $n=1$, using the formula $\Gamma(z+1)=z \Gamma(z)$ and simplifying the above result we obtain the following inequality:

$$
\left|a_{1}\right|^{2}+\left|b_{1}\right|^{2} \geq B_{1}(K):=2-2^{2(1-1 / K)\left(2+2^{5 / 4}\right)} \frac{2 K^{2} \Gamma\left(\frac{2}{K}\right)}{(K+1) \Gamma^{2}\left(\frac{1}{K}\right)} .
$$

By computation we know that $B_{1}(K)$ is a decreasing function of $K$ and satisfies

$$
\lim _{K \rightarrow 1^{+}} B_{1}(K)=1
$$

The above estimate is sharp. Consider the conformal mapping $w(z)=e^{i x} z$, where $x \in \mathbb{R}$ is a real number. Then we have $\left|a_{1}\right|+\left|b_{1}\right|=1$.

For $n \geq 2$, we have

$$
\begin{aligned}
& \Gamma\left(1+\frac{1}{K}-n\right)=\frac{\Gamma\left(\frac{1}{K}\right)}{\left(1+\frac{1}{K}-n\right)\left(2+\frac{1}{K}-n\right) \cdots\left(\frac{1}{K}-1\right)}=\frac{(-1)^{n-1} \Gamma\left(\frac{1}{K}\right)}{\left(n-1-\frac{1}{K}\right) !} \\
& \Gamma\left(1+\frac{1}{K}+n\right)=\left(n+\frac{1}{K}\right)\left(n+\frac{1}{K}-1\right) \cdots\left(\frac{1}{K}\right) \Gamma\left(\frac{1}{K}\right)=\frac{1}{K} \Gamma\left(\frac{1}{K}\right)\left(n+\frac{1}{K}\right) !
\end{aligned}
$$

then

$$
\left|a_{n}\right|^{2}+\left|b_{n}\right|^{2} \geq \chi(K)+\frac{2^{2(1-1 / K)\left(2+2^{5 / 4}\right)} \Gamma\left(1+\frac{2}{K}\right)\left(n-1-\frac{1}{K}\right) !}{\Gamma^{2}\left(\frac{1}{K}\right)\left(n+\frac{1}{K}\right) \frac{1}{K}\left(n-1+\frac{1}{K}\right) !}:=B_{n}(K) .
$$

By calculating we see that $\chi(K)$ is a decreasing function of $K$ with $\chi(1)=0$. The function $B_{n}(K)$ is a continuous function of $K$ with $\lim _{K \rightarrow 1^{+}} B_{n}(K)=\frac{2}{(n+1) n(n-1)}$. This implies that $B_{n}(K)>0$ holds for all $n \geq 2$ and some $K>1$.

The proof is completed.

Remark 1 By computation we obtain

$$
B_{1}(K)>\frac{27}{4 \pi^{2}}
$$


for all $1 \leq K \leq 1.05174$. This shows that under the additional assumption that $w$ is a $K$-quasiconformal mapping, the lower bound of the inequality (3) can be improved.

By the definition of the Gamma function we see that $\Gamma(-n)=\infty$ holds for all nonnegative integer numbers $n$. According to the proof of Theorem 1 we know that for all $n \geq 2$, $\lim _{K \rightarrow 1^{+}} \Gamma\left(1+\frac{1}{K}-n\right)=\infty$. Therefore

$$
\lim _{K \rightarrow 1^{+}} B_{n}(K)=0
$$

holds for all $n \geq 2$.

Let $t=0$ in equation (21). Then we have $\sum_{n=1}^{\infty}\left(\left|a_{n}\right|^{2}+\left|b_{n}\right|^{2}\right)=1$. The sharp coefficient estimate of $a_{1}$ and $b_{1}$ shows that if $K \rightarrow 1^{+}$then $\left|a_{1}\right|^{2}+\left|b_{1}\right|^{2} \geq B_{1}(K) \rightarrow 1$. This shows that under the assumptions of Theorem 1 if additionally $w(z)$ is a conformal self-mapping of $\mathbb{D}$ satisfying $w(0)=0$, then all the coefficients $b_{n}$ for $n \geq 1$ and $a_{n}$ for $n \geq 2$ are zeros and $\left|a_{1}\right|=1$, that is, $w(z)=e^{i \theta} z$ for some $\theta \in \mathbb{R}$.

Remark 2 In [1] the authors showed that an asymptotically sharp inequality holds for all $z$ in $\mathbb{D}$. Our Theorem 1 , however, gives an estimate at $z=0$ only. In this sense, Theorem 1 partly improves the former results.

Theorem 2 shows that $n^{2}\left(\left|a_{n}\right|^{2}+\left|b_{n}\right|^{2}\right)$ is less than or equal to a positive number determined by $K$.

Theorem 2 Under the assumption of Theorem 1, the coefficients of $w(z)$ satisfy the following inequality:

$$
\left|a_{n}\right|^{2}+\left|b_{n}\right|^{2} \leq \frac{16}{n^{2} \pi^{2}} K^{6 K} 2^{5(K-1 / K)}, \quad n=1,2, \ldots
$$

Proof For every $z=r e^{i \theta} \in \mathbb{D}$,

$$
w\left(r e^{i \theta}\right)=\sum_{n=1}^{\infty} a_{n} r^{n} e^{i n \theta}+\sum_{n=1}^{\infty} \bar{b}_{n} r^{n} e^{-i n \theta}
$$

hence

$$
\begin{aligned}
& a_{n} r^{n}=\frac{1}{2 \pi} \int_{0}^{2 \pi} w\left(r e^{i \theta}\right) e^{-i n \theta} d \theta, \quad n=1,2, \ldots, \\
& \bar{b}_{n} r^{n}=\frac{1}{2 \pi} \int_{0}^{2 \pi} w\left(r e^{i \theta}\right) e^{i n \theta} d \theta, \quad n=1,2, \ldots
\end{aligned}
$$

For every $n$ we set $a_{n}=\left|a_{n}\right| e^{i \alpha_{n}}, b_{n}=\left|b_{n}\right| e^{i \beta_{n}}$, and $\theta_{n}=\frac{\alpha_{n}+\beta_{n}}{2 n}$. Then

$$
\begin{aligned}
\left(\left|a_{n}\right|+\left|b_{n}\right|\right) r^{n} & =\left|\frac{1}{2 \pi} \int_{0}^{2 \pi} w\left(r e^{i \theta}\right)\left[e^{-i \alpha_{n}} e^{-i n \theta}+e^{i \beta_{n}} e^{i n \theta}\right] d \theta\right| \\
& =\left|\frac{1}{2 \pi} \int_{0}^{2 \pi} w\left(r e^{i \theta}\right)\left[e^{-i n\left(\theta+\theta_{n}\right)}+e^{i n\left(\theta+\theta_{n}\right)}\right] d \theta\right| \\
& =\left|\frac{1}{\pi} \int_{0}^{2 \pi} w\left(r e^{i \theta}\right) \cos n\left(\theta+\theta_{n}\right) d \theta\right|
\end{aligned}
$$


Integrating by parts we have

$$
\left(\left|a_{n}\right|+\left|b_{n}\right|\right) r^{n}=\left|\frac{1}{n \pi} \int_{0}^{2 \pi} w_{\theta}\left(r e^{i \theta}\right) \sin n\left(\theta+\theta_{n}\right) d \theta\right| .
$$

In Theorem 2.8 of [10], Kalaj proved that the radial limits of $w_{\theta}$ and $w_{r}$ exist almost everywhere and

$$
\lim _{r \rightarrow 1^{-}} \partial_{\theta} w\left(r e^{i \theta}\right)=\frac{d f\left(e^{i \theta}\right)}{d \theta}
$$

for almost every $z=e^{i \theta} \in \mathbf{T}$. Here $f$ is the boundary function of $w$. Hence, letting $r \rightarrow 1^{-}$ and using (13), (23) we see that

$$
\left|a_{n}\right|+\left|b_{n}\right| \leq \frac{1}{n \pi} \int_{0}^{2 \pi}\left|f^{\prime}\left(e^{i \theta}\right)\right|\left|\sin n\left(\theta+\theta_{n}\right)\right| d \theta=\frac{4 K^{3 K} 2^{5(K-1 / K) / 2}}{n \pi} .
$$

It shows that $\left|a_{n}\right|^{2}+\left|b_{n}\right|^{2} \leq\left(\left|a_{n}\right|+\left|b_{n}\right|\right)^{2} \leq \frac{16 K^{6 K} 2^{5(K-1 / K)}}{n^{2} \pi^{2}}:=A_{n}(K)$.

The proof is completed.

\section{Competing interests}

The author declares that she has no competing interests.

\section{Acknowledgements}

The author of this work is supported by NNSF of China (Nos. 11301195, 11501220) and the China Scholarship Council and a research foundation of Huaqiao University (Project No. 2014KJTD14). The author would like to express her appreciation to Professor Jian-Feng Zhu and the referee for their helpful advice.

Received: 17 November 2015 Accepted: 24 February 2016 Published online: 02 March 2016

\section{References}

1. Partyka, D, Sakan, K: On an asymptotically sharp variant of Heinz's inequality. Ann. Acad. Sci. Fenn., Math. 30, 167-182 (2005)

2. Zhu, JF, Zeng, X: Estimate for Heinz inequality in the small dilatation of harmonic mappings. J. Comput. Anal. Appl. 13, 1081-1087 (2011)

3. Lewy, H: On the non-vanishing of the Jacobian in certain one-to-one mappings. Bull. Am. Math. Soc. 42, 689-692 (1932)

4. Heinz, E: Über die Lösungen der Minimalflächengleichung. Nachr. Akad. Wiss. Gött. Math.-Phys. Kl., $51-56$ (1952)

5. Hall, PR: On an inequality of E. Heinz. J. Anal. Math. 42, 185-198 (1982)

6. Qiu, S, Ren, L: Sharp estimates for Hübner's upper bound function with applications. Appl. Math. J. Chin. Univ. Ser. B 25, 227-235 (2010)

7. Duren, P: Harmonic Mappings in the Plane. Cambridge University Press, New York (2004)

8. Pavlović, M: Boundary correspondence under harmonic quasiconformal homeomorphisms of the unit disk. Ann. Acad. Sci. Fenn., Ser. A I Math. 27, 365-372 (2002)

9. Partyka, D, Sakan, K: On bi-Lipschitz type inequalities for quasiconformal harmonic mappings. Ann. Acad. Sci. Fenn., Math. 32, 579-594 (2007)

10. Kalaj, D: Quasiconformal harmonic functions between convex domains. Publ. Inst. Math. 76(90), 3-20 (2004) 